\title{
Liquid Scintillators with Near Infrared Emission Based on Organoboron Conjugated Polymers
}

Kazuo Tanaka*a ${ }^{\text {, }}$ Takayuki Yanagida ${ }^{\mathrm{b}}$, Honami Yamane ${ }^{\mathrm{a}}$, Amane Hirose ${ }^{\mathrm{a}}$, Ryousuke Yoshii ${ }^{\mathrm{a}}$, Yoshiki Chujo*a

${ }^{a}$ Department of Polymer Chemistry, Graduate School of Engineering, Kyoto University, Katsura, Nishikyo-ku, Kyoto 615-8510, Japan

${ }^{b}$ Graduate School of Materials Science, Nara Institute of Science and Technology, 8916-5 TakayamaCho, Ikoma, Nara 630-0192, Japan

Y.C., chujo@chujo.synchem.kyoto-u.ac.jp

K.T., kazuo123@chujo.synchem.kyoto-u.ac.jp

\section{KEYWORDS}

Organoboron complex, conjugated polymer, scintillation, near infrared emission 


\section{ABSTRACT}

The organic liquid scintillators based on the emissive polymers are reported. A series of conjugated polymers containing organoboron complexes which show the luminescence in the near infrared (NIR) region were synthesized. The polymers showed good solubility in common organic solvents. From the comparison of the luminescent properties of the synthesized polymers between optical and radiation excitation, similar emission bands were detected. In addition, less significant degradation was observed. These data propose that the organoboron conjugated polymers are attractive platforms to work as an organic liquid scintillator with the emission in the NIR region. 
Scintillators which present a luminescence by absorbing radiation are versatile materials not only for quantifying an environmental radiation dose but also for using in the modern medical instruments. Development of higher-performance scintillators has been still required for advanced radiation monitoring such as highly-sensitive detection, precise quantification and discrimination of the radiation species. ${ }^{1}$ Most of conventional scintillators are composed of inorganic crystals, and the preparation for the crystals with low cost has been radically explored. ${ }^{2}$ Organic polymers are intrinsically cheap, light and flexible. In particular, polymers have high processability and film-formability which are useful properties for the preparation of the plastic-type sensors. Moreover, color and other optical properties can be readily tuned by altering the chemical structures, the introduction of element blocks which are consisted of heteroatom-containing functional units or groups and the conjugation with various comonomers by employing an alternating copolymer structure according to the preprogrammed design. ${ }^{3}$ Thus, polymer-based materials are attractive candidates as a platform for developing new series of scintillators. However, even the number of all organic scintillators is much fewer than those of inorganic ones. Although polymers have been used as a matrix in hybrid material-based scintillators or mixture systems, the polymer-based scintillator with the single chemical component is still very few., Especially, the scintillation emissions were observed in the different wavelength regions from the photoluminescence spectra. This fact means that it is still difficult to obtain the scintillation emission at the desired emission wavelength. In addition, there are much room to explore systematic information on the relationships between chemical structure and scintillation ability. To probe the feasibility of organic materials as a scintillator, fundamental information on the optical properties should be needed. The comparison study on the luminescence by the photon excitation and the radiation irradiation should be essential for receiving expected scintillators based on the molecular design.

Because of the high permeability through air and aqueous media involving vital organs, the NIR is applied in the sensing technology. ${ }^{6}$ The probes with the NIR emission can present the signal at the relatively-deeper spot inside bodies comparing to commodity fluorescence materials. Therefore, the 
development of efficient NIR-emissive materials is still a topic with high relevance. ${ }^{7}$ Versatility of organoboron conjugated polymers have been revealed from a series of the recent works as a typical example of element blocks. ${ }^{3,8}$ Particularly, various NIR-emissive molecules and polymers have been reported. ${ }^{9}$ For example, it was found that the boron di(iso)indomethene-containing polymers presented strong emission in the NIR region. ${ }^{10}$ Especially, the sharp emission bands were observed in the spectra. These properties are feasible for obtaining vivid color from the display and for improving the sensitivity in the bioimaging usages. As another instance, the NIR-emissive boron dipyrromethene (BODIPY) was introduced into the polymer main-chains. ${ }^{9,11}$ Since the resulting polymers also showed high electroncarrier ability, the application of these polymers to the electroluminescent devices progresses. ${ }^{11}$ Based on the flexibility in the molecular design and various functions derived from a variety of chemical structures, unique characteristics were obtained. Thus, it can be expected that organoboron polymers are a promising platform for producing a new series of advanced scintillators.

Herein, the NIR-emissive organoboron conjugated polymers were synthesized. Particularly, we aimed to obtain the similar emission spectra from the polymer solutions with both photo- and X-ray excitations. To fulfill the purpose of this research, the comparison study of the optical properties of the synthesized conjugated polymers with the optical and radiation excitation was performed. Emission efficiencies and the decay times were evaluated. In addition, the stability was also investigated. The structure-property relationship for the application to organic scintillators is mainly discussed. 
It has been reported that the tetraphenyl-substituted BODIPY and aza-BODIPY derivatives showed bright emissions in deep-red and NIR regions due to the narrow band gaps (Scheme 1). ${ }^{9,11}$ At the meso position in the BODIPY derivatives, the lowest unoccupied molecular orbital (LUMO) was often delocalized. ${ }^{12}$ Thereby, by replacing the $\mathrm{CH}$ at the meso position to $\mathrm{N}$, only the energy level of LUMO can be lowered, leading to the red-shifted emission. ${ }^{9,11}$ In this study, we aimed to evaluate the influence of this modification of chemical structures on the scintillation ability. The sharp emission spectra are advantageous for a sensing material. Especially, it was presented from the recent paper that these BODIPYs can have high electron-carrier abilities. ${ }^{11}$ Thus, it is presumed that the side-reactions or the deactivation of the excitons generated by the higher-energy light irradiation might be suppressed, resulting in efficient scintillation. However, free BODIPYs would readily form aggregation even in the diluted solution state. Therefore, the chemical modification to maintain solubility should be needed to keep the homogeneous solution state. One of solves to improve solubility is the formation of the polymers. Particularly, by introducing into alternating polymers, the tunability of the electronic states as well as the enhancement of solubility can be expected. Thus, we designed the BODIPY-containing alternating polymers with alkyl-substituted fluorene which has a superior property for enhancing solubility and light-absorption ability.

Scheme 1. Synthetic scheme of the polymers ${ }^{a}$

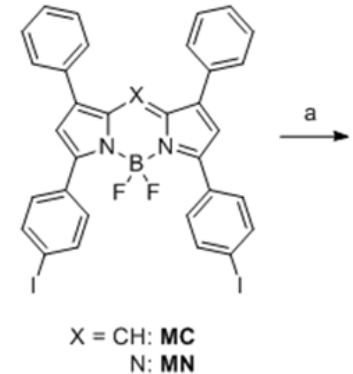

N: MN

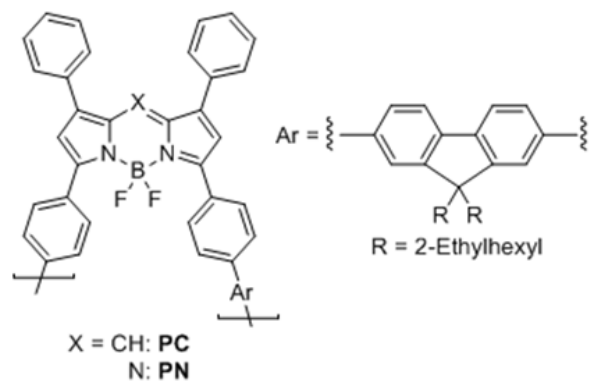

\footnotetext{
${ }^{a}$ Reagents and conditions: (a) [9,9-Bis(2-ethylhexyl)-9H-fluorene-2,7-diyl]bisboronic acid, $\operatorname{Pd}_{2}\left(\mathrm{dba}_{3}\right.$, S-Phos, cesium carbonate, toluene, $80^{\circ} \mathrm{C}, 3 \mathrm{~d}$, (PC: $96 \%$, PN: $\left.85 \%\right)$.
} 
The monomers were prepared according to the previous report. ${ }^{11}$ The polymerization with fluorenyldiboronic acid via Suzuki-Miyaura coupling is illustrated in Scheme 1. To maintain the solubility of the products, the 9-position at the center of the fluorene unit was modified with the alkyl chains. After the reaction and reprecipitation from methanol to remove the metal species and small molecules derived from the monomers, the products were obtained. A series of measurements were performed to characterize the products (Table 1). The number-average molecular weight $\left(M_{\mathrm{n}}\right)$ and polydispersity index $\left(M_{\mathrm{w}} / M_{\mathrm{n}}\right)$ of the polymer determined from GPC analysis were evaluated, and the values of degree of polymerization (DP) were calculated from the obtained molecular weights. The polymer showed moderate solubilities in conventional organic solvents such as chloroform, dichloromethane, and THF. From the ${ }^{1} \mathrm{H}$ and ${ }^{11} \mathrm{~B}$ NMR spectra, similar signal patterns involving azaBODIPY and the fluorene units in the polymer were obtained. We concluded that the polymers should have the designed structures as we expected.

Table 1. Physical properties of the polymers ${ }^{a}$

\begin{tabular}{ccc}
\hline & PC & PN \\
\hline$M_{\mathrm{n}}$ & 4,300 & 7,700 \\
$M_{\mathrm{w}} / M_{\mathrm{n}}$ & 2.0 & 2.0 \\
$\mathrm{DP}^{b}$ & 4.9 & 8.7 \\
Yield (\%) $^{c}$ & 85 & 96 \\
\hline${ }^{a}$ Estimated by size-exclusion chromatography (SEC) based on polystyrene standards in chloroform. & ${ }^{b}$ Degree of polymerization estimated \\
by number-average molecular weight. & ${ }^{c}$ Isolated yields after precipitation.
\end{tabular}

The optical properties of the synthesized polymers were investigated. Figure 1 shows the UV-vis absorption spectra in toluene. The results are summarized in Table 2. The largest absorption bands with the peaks at $610 \mathrm{~nm}$ and $703 \mathrm{~nm}$ assigned to the $\pi-\pi^{*}$ transitions at each complex were observed from PC and PN, respectively. Comparing the peak positions of the absorption bands to those of the monomers (MC: $579 \mathrm{~nm}, \mathbf{M N}$ : $664 \mathrm{~nm}$ ), the polymers provided the absorption in the red-shifted regions. ${ }^{11}$ These results indicate that the main-chain conjugation should be expanded in the ground state. 
In addition, the similar peak shapes were observed from the solutions. The unexpected inter-chain interaction or aggregation could be suppressed in the samples. For the measurements with the optical excitation, the samples were irradiated with the light at the absorption maxima.

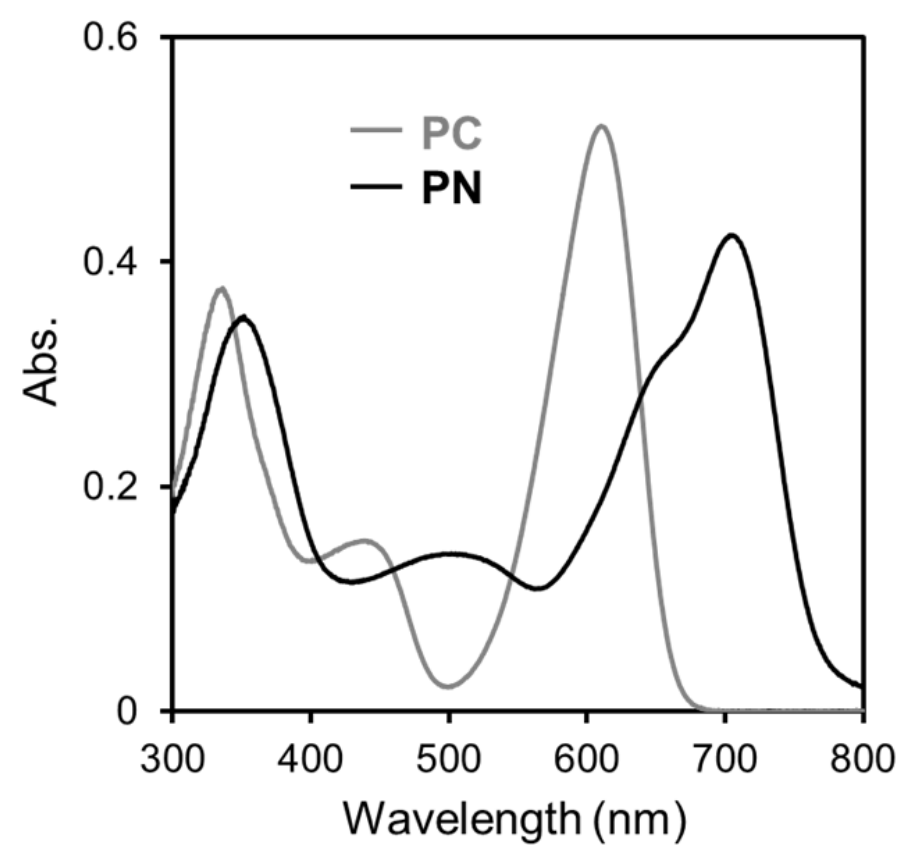

Figure 1. UV-vis absorption spectra of the polymers in toluene $\left(1.0 \times 10^{-5} \mathrm{M}\right)$.

Table 2. Photophysical properties of the polymers ${ }^{a}$

\begin{tabular}{ccc}
\hline & PC & PN \\
\hline$\lambda_{\text {Abs,max }}(\mathrm{nm})$ & 610 & 703 \\
$\varepsilon\left(\mathrm{M}^{-1} \mathrm{~cm}^{-1}\right)$ & 52,000 & 42,000 \\
$\lambda_{\mathrm{PL}, \max }(\mathrm{nm})^{b}$ & 654 & 750 \\
$\Phi_{\mathrm{PL}}{ }^{b}$ & 0.47 & 0.028 \\
$\lambda_{\text {X-ray,max }}(\mathrm{nm})$ & 662 & 752 \\
$\tau_{\mathrm{PL}}(\mathrm{ns})^{d}$ & $1.64(0.04), 4.23(0.96)$ & 1.92 \\
$\tau_{\text {X-ray }}(\mathrm{ns})$ & $0.46(0.90), 2.91(0.10)$ & $0.36(0.88), 2.22(0.12)$ \\
\hline
\end{tabular}

\footnotetext{
${ }^{a}$ Measured in toluene $\left(1.0 \times 10^{-5} \mathrm{M}\right){ }^{b}$ Measured with the excitation light at the maximum peak wavelength of absorption. ${ }^{c}$ Absolute quantum yield. ${ }^{d}$ Measured with the excitation light at $375 \mathrm{~nm}$. Parentheses mean the proportions of each element in the decay curves.
}

Photoluminescence (PL) spectra were gathered with the solutions containing the synthesized polymers in toluene (Figure 2). The optical properties are summarized in Table 2. Typical spectra were obtained from the measurements. Emission bands with the peaks at $654 \mathrm{~nm}$ and $750 \mathrm{~nm}$ attributable to the $\mathrm{S}_{0}-\mathrm{S}_{1}$ $\left(\pi-\pi^{*}\right)$ transition were obtained from PC and PN, respectively. These data mean that the polymers 
should be NIR-emissive materials. The peak shifts to the longer wavelength regions than those in each monomer were also observed (MC: $613 \mathrm{~nm}$, MN: $694 \mathrm{~nm}$ ). ${ }^{11}$ These data indicate the elongation of the conjugation system through the polymer main-chains in the excited state. The emission lifetimes were measured (Table 2). The kinetics of the emission were evaluated from the time-resolved luminescent spectra. The lifetimes were calculated from the fitting curves to the emission decay. It was confirmed that the emissions of the synthesized polymers should be assigned as fluorescence.

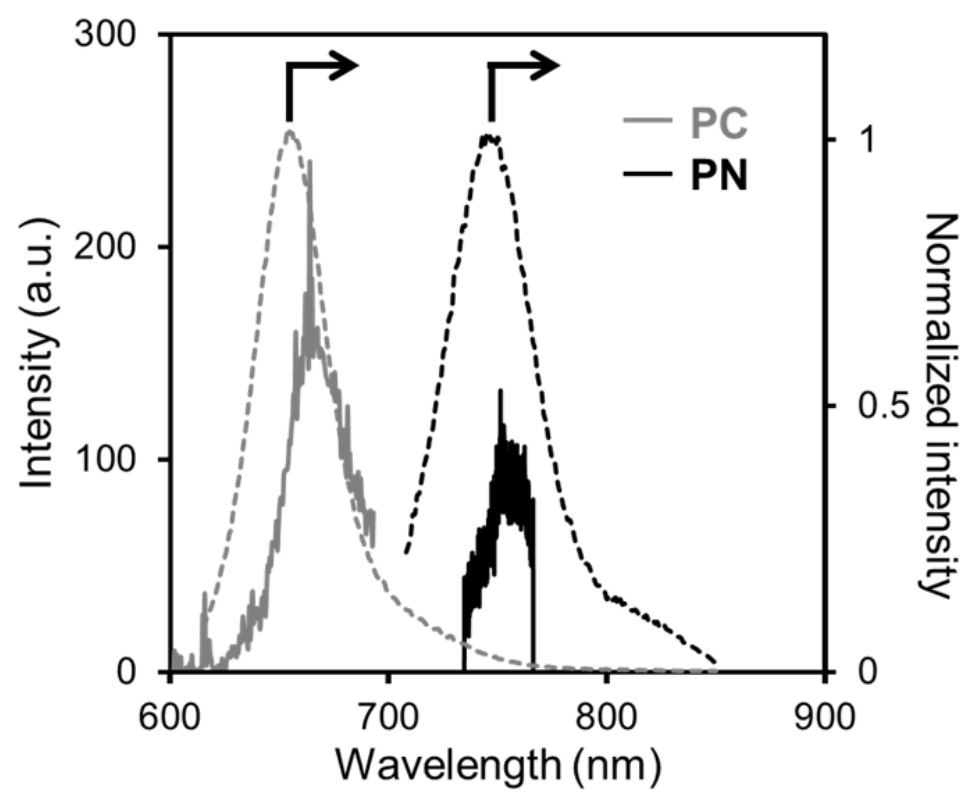

Figure 2. Emission spectra of the polymers in toluene $\left(1.0 \times 10^{-5} \mathrm{M}\right)$ with the excitation light at the wavelength of the absorption maxima (dot line) and X-ray (solid line).

Scintillation emission spectrum under $\mathrm{X}$-ray irradiation is shown in Figure 2. X-ray induced radioluminescence spectra of the polymers were evaluated by using our original setup. ${ }^{13-17}$ The details are described in the Supporting Information. All samples containing $10^{-5} \mu \mathrm{M}$ in toluene $(10 \mathrm{~mL})$ were sealed into a glass columnar bottle (height: $4.5 \mathrm{~cm}$, bottom diameter $1.5 \mathrm{~cm}$ ), and X-ray was irradiated from the bottom. The emission bands were observed in the deep red and NIR regions. These data 
represent that the synthesized polymers should have the scintillation ability in the solution states. In particular, the emission peaks were obtained at $662 \mathrm{~nm}$ and $752 \mathrm{~nm}$ from PC and PN, respectively. It is noteworthy that the similar spectra were obtained with the PL spectra. The scintillation decay time profile of the synthesized polymers was measured. The time profiles were well reproduced by a double exponential assumption. Deduced decay times are listed in Table 2 and showed good correlations to those from the PL data. These results strongly support that the scintillation from the synthesized polymers should originate from the $\mathrm{S}_{0}-\mathrm{S}_{1}\left(\pi-\pi^{*}\right)$ transition. In other words, the scintillation properties can be estimated by the PL spectra. In addition, to evaluate the durability against the X-ray irradiation, we measured the PL spectra again, and similar spectra were obtained from each polymer solution. This result means that the degradation in both polymer samples hardly occurred after the radiation irradiation (360 Gy). It is implied that the synthesized polymers could have stability enough for the application as a scintillator. So far, there are very few scintillators involving inorganic materials with the emission in the NIR regions. Thereby, it is difficult to directly compare and quantitatively evaluate the scintillation ability to the previous materials at this stage. It is implied that our materials can be used as a standard for evaluating the scintillation ability in the NIR region.

In conclusion, the conjugated polymers with the similar emission spectra via both photo- and X-ray excitations were demonstrated in this study. The fundamental information on the scintillation abilities of the organoboron NIR-emissive polymers is also presented. It is shown that the organoboron-containing conjugated polymers can work as a liquid scintillator in the organic solvent. It should be emphasized that the scintillation properties showed good correlation to the luminescence by the optical excitation. These facts represent that an expected scintillation ability could be obtained according to the preprogrammed design. Furthermore, organoboron materials involving polymers are paid attention as a neutron-selective scintillator due to the intrinsic reactivity of boron to neutron. Therefore, our findings described here might be useful not only for developing organic-based advanced functional scintillators but also for constructing neutron-selective sensors. 


\section{Acknowledgements}

This work was partially supported by the SEI Group CSR Foundation (for K.T.), "the Adaptable and Seamless Technology Transfer Program" through target-driven R\&D, Japan Science and Technology Agency (JST) and a Grant-in-Aid for Scientific Research on Innovative Areas "New Polymeric Materials Based on Element-Blocks (No.2401)" (24102013) of The Ministry of Education, Culture, Sports, Science, and Technology, Japan. 


\section{References and notes}

1. (a) Nikl, M.; Yoshikawa, A.; Kamada, K.; Nejezchleb, K.; Stanek, C. R.; Mares, J. A.; Blazek, K. Prog. Cryst. Growth Charact. Mater. 2013, 59, 47-72. (b) Li, J.-G.; Sakka, Y. Sci. Technol. Adv. Mater. 2015, 16, 014902. (c) Yamamoto, Y.; Tasaki, Y.; Kuwada, Y.; Ozawa, Y.; Katayama, A.; Kanemaki, Y.; Enokido, K.; Nakamura, S.; Kubouchi, K.; Morita, S.; Noritake, M.; Nakajima, Y.; Inoue, T. Ann. Nucl. Med. 2013, 27, 795-801.

2. (a) Masai, H.; Hino, Y.; Yanagida, T.; Fujimoto, Y.; Tokuda, Y. Opt. Mater. Express 2015, 5, 617-622. (b) Masai, H.; Hino, Y.; Yanagida, T.; Fujimoto, Y. Opt. Mater. 2015, 42, 381-384. (c) Yanagida, T.; Fujimoto, Y.; Koshimizu, M.; Fukuda, K. J. Lumin. 2015, 157, 293-296.

3. Chujo, Y.; Tanaka, K. Bull. Chem. Soc. Jpn 2015, 88, 633-643.

4. (a) Koshimizu, M.; Kitajima, H.; Iwai, T.; Asai, K. Jpn. J. Appl. Phys. 2008, 47, 5717-5719. (b) Bertrand, G. H. V.; Hamel, M.; Normand, S.; Sguerra, F. Nucl. Instrum. Methods A 2015, 776, 114-128. (c) Bertrand, G. H. V.; Hamel, M.; Sguerra, F. Chem. Eur. J. 2014, 20, 15660-15685. (d) Sun, Y.; Koshimizu, M.; Yahaba, N.; Nishikido, F.; Kishimoto, S.; Haruki, R.; Asai, K. Appl. Phys. Lett. 2014, 104, 174104. (e) Sguerra, F.; Marion, R.; Bertrand, G. H. V.; Coulon, R.; Sauvageot, É.; Daniellou, R.; Renaud, J.-L.; Gaillard, S.; Hamel, M. J. Mater. Chem. C 2014, 2, 6125-6133.

(f) Bertrand, G. H. V.; Sguerra, F.; Dehé-Pittance, C.; Carrel, F.; Coulon, R.; Normand, S.; Barat, E.; Dautremer, T.; Montagu, T.; Hamel, M. J. Mater. Chem. C 2014, 2, 7304-7312. (g) Nakamura, H.; Shirakawa, Y.; Kitamura, H.; Yamada, T.; Shidara, Z.; Yokozuka, T.; Nguyen, P.; Takahashi, T.; Takahashi, S. Radiat. Meas. 2013, 59, 172-175.

5. (a) Kamaya, E.; Matsumoto, F.; Kondo, Y.; Chujo, Y.; Katagiri, M. Nucl. Instrum. Methods A 2004, 529, 329-331. (b) Nakamura, H.; Shirakawa, Y.; Kitamura, H.; Sato, N.; Shinji, O.; Saito, K.; Takahashi, S. Appl. Phys. Lett. 2013, 103, 161111. (c) Adadurov, A. F.; Yelyseev, D. A.; Titskaya, V. D.; Lebedev, V. N.; Zhmurin, P. N. Radiat. Meas. 2011, 46, 498-502.

6. Weissleder, R. Nat. Biotechnol. 2001, 19, 316-317. 
7. (a) Yen, S. K.; Janczewski, D.; Lakshmi, J. L.; Dolmanan, S. B.; Tripathy, S.; Ho, V. H. B.; Vijayaragavan, V.; Hariharan, A.; Padmanabhan, P.; Bhakoo, K. K.; Sudhaharan, T.; Ahmed, S.; Zhang, Y.; Selvan, S.T. ACS Nano 2013, 7, 6796-6805. (b) Pansare, V. J.; Hejazi, S.; Faenza, W. J.; Prud'homme, R. K. Chem. Mater. 2012, 24, 812-827.

8. Tanaka, K.; Chujo, Y. Macromol. Rapid Commun. 2012, 33, 1235-1255.

9. Yoshii, R.; Nagai, A.; Chujo, Y. J. Polym. Sci. Part A: Polym. Chem. 2010, 48, 5348-5356.

10. Yoshii, R.; Nagai, A.; Tanaka, K.; Chujo, Y. J. Polym. Sci. Part A: Polym. Chem. 2013, 51, 17261733.

11. Yoshii, R.; Yamane, H.; Nagai, A.; Tanaka, K.; Taka, H.; Kita, H.; Chujo, Y. Macromolecules 2014, 47, 2316-2323.

12. (a) Gresser, R.; Hartmann, H.; Wrackmeyer, M.; Leo, K.; Riede, M. Tetrahedron 2011, 67, 7148-7155. (b) Tanaka, K.; Yamane, H.; Yoshii, R.; Chujo, Y. Bioorg. Med. Chem. 2013, 21, $2715-2719$.

13. Yanagida, T.; Kamada, K.; Fujimoto, Y.; Yagi, H.; Yanagitani, T. Opt. Mater. 2013, 35, $2480-2485$.

14. Yanagida, T.; Fujimoto, Y.; Fukuda, K.; Chani, V. Nucl. Instrum. Methods A 2013, 729, 58-63.

15. Yanagida, T.; Fujimoto, Y.; Yamaji, A.; Kawaguchi, N.; Kamada, K.; Totsuka, D.; Fukuda, K.; Yamanoi, K.; Nishi, R.; Kurosawa, S.; Shimizu, T.; Sarukura, N. Radiat. Meas. 2013, 55, 99-102.

16. Yanagida T.; Fujimoto Y.; Yoshikawa A.; Yokota Y.; Kamada K.; Pejchal J.; Kawaguchi N.; Fukuda K.; Uchiyama K.; Mori K.; Kitano K.; Nikl M.; Appl. Phys. Exp. 2010, 3, 056202.

17. Yanagida, T.; Fujimoto, Y.; Ito, T.; Uchiyama, K.; Mori, K. Appl. Phys. Exp. 2014, 7, 062401. 